\section{Gemcitabin und Pemetrexed bieten verbesserte Perspektiven bei thorakalen Tumoren}

\begin{abstract}
Mit mehr als einer Million Neudiagnosen weltweit pro Jahr handelt es sich beim nichtkleinzelligen Bronchialkarzinom (NSCLC) um einen der häufigsten Tumoren. Galten die therapeutischen Optionen lange Zeit als sehr begrenzt, sind in den letzten Jahren deutliche Fortschritte festzustellen. Moderne Zweier-Kombinationen auf Platin-Basis und eine Kombination mit effektiven, neueren Substanzen wie Gemcitabin $\left(\right.$ Gemzar $\left.^{\circledR}\right)$ verlängern in der First-line-Therapie die Überlebenszeit, lindern tumorassoziierte Beschwerden und steigern die Lebensqualität. Auch in der Second-line-Therapie steht mit Pemetrexed (Alimta ${ }^{\circledR}$ ) eine effektive und verträgliche Therapieoption zur Verfügung. Dieser Multi-Target-Enzym-Inhibitor hat zudem in Kombination mit Cisplatin beim malignen Pleuramesotheliom einen Durchbruch bewirkt. Durch diese Kombination gilt diese seltene, aber zunehmende Tumorentität unter den thorakalen Tumoren endlich als behandelbar und einer Chemotherapie zugänglich.
\end{abstract}

\section{Deutliche Fortschritte in allen Behandlungssituationen}

Die aktuellen ermutigenden Entwicklungen betreffen sowohl die fortgeschrittenen Stadien als auch die adjuvanten Ansätze beim NSCLC. «Mittlerweile können wir nicht nur Patienten in der First-line-Therapie beim fortgeschrittenen Bronchialkarzinom effektive Therapieoptionen anbieten, sondern auch noch bei weiterer Krankheitsprogression eine hohe Effektivität und Linderung der Symptome erreichen», berichtete Professor Michael Thomas von der Thoraxklinik Heidelberg. Darüber hinaus ist aktuell die Etablierung der adjuvanten Chemotherapie nach kompletter Resektion von großer Bedeutung, die zu einer Steigerung der Überlebensrate führt. Inwieweit Platin-haltige Kombinationen mit modernen Substanzen wie Gemcitabin einen nochmaligen Fortschritt bedingen, müssen weitere Untersuchungen abklären.

\section{Gemcitabin mit deutlichen Vorteilen in der First-line-Therapie}

In der Behandlung des fortgeschrittenen NSCLC (Stadium IIIb/IV) gilt die Chemotherapie schon längere Zeit als Behandlungsbasis, da sie - vor allen Dingen in Kombination mit modernen Substanzen wie Gemcitabin zu einem verlängerten Überleben und weniger Symptomen führt. «In Anbetracht der Tatsache, dass 3 von 4 Patienten schon bei Diagnosestellung eine fortgeschrittene Erkrankung haben, sind die Vorteile der Therapie wie längeres und besseres Überleben von herausragender klinischer Relevanz», bestätigte Dr. Wolfgang Schütte, Halle. Standard sind Zweierkombinationen auf Platin-Basis. Dreierkombinationen bewirken laut Datenlage meist nur ein geringfügig höheres Ansprechen, welches meist von einer höheren Toxizität begleitet ist. Laut der Metaanalyse von Ardezzoni et al. [1] bietet sich als Grundlage der Therapien Cisplatin an. Bei Patienten mit einem schlechten Performance-Status oder Komorbiditäten kann Carboplatin als Alternative verwendet werden. Gemcitabin hat sich in Studien als Kombinationspartner von Cis- und Carboplatin mit einer hohen Effektivität bei vertretbaren hämatologischen Nebenwirkungen hervorgetan. Dieser Fakt wird durch eine Metaanalyse nachhaltig unterstützt. Im Rahmen dieser Untersuchung konnte gezeigt werden, dass Platin-basierte Kombinationen mit Gemcitabin anderen Platin-Kombinationen beim NSCLC hinsichtlich der Effektivität überlegen sind [2]. Es ergab sich ein statistisch signifikanter Überlebensvorteil für Platin/Gemcitabin gegenüber den anderen Platin-Kombinationen (1-JahresÜberleben 38,9 vs. $35 \%$; Abb. 1.)

\section{Individuell und zielgerichtet behandeln}

Schütte unterstreicht, dass eine Chemotherapie heute keine Frage des Alters mehr ist: «Früher wurden über 65-jährige nicht mehr therapiert, heute können wir für ältere Patienten auch auf Gemcitabin-haltige Kombinationen zurückgreifen.» Bei Patienten mit sehr schlechtem Allgemeinzustand ist zur Symptomlinderung eine wirksame Gemcitabin-Monotherapie in Betracht zu ziehen. Neuere Behandlungsoptionen orientieren sich an den molekularen Grundlagen des NSCLC. So deuten aktuelle Arbeiten darauf hin, dass bei erhöhter Expression des DNA-Reparaturenzyms ERCC-1 Cisplatin seine Wirkung einbüßt. Dann könnten Platin-freie Kombinationen wie zum Beispiel Gemcitabin/Vinorelbin zum Einsatz kommen. Obwohl es sich laut des Lungenkrebsexperten bei diesen Studien noch um Phase-II-Untersuchungen handelt, könnten diese in Zukunft jedoch dazu beitragen, einen gezielteren Einsatz der verschiedenen Chemotherapieoptionen beim Lungenkarzinom zu ermöglichen.

\section{Pemetrexed als effektiver, verträglicher Standard in der Second-line-Therapie}

Obwohl auch die Leitlinien der Amerikanischen Gesellschaft für klinische Onkologie (ASCO) eine Second-line-Therapie beim NSCLC empfehlen, wird diese noch viel zu selten umgesetzt, bemängelte Professor Dr. Axel Hanauske, Hamburg. Das ist umso unverständlicher, als mit Pemetrexed eine Substanz zur Verfügung steht, die gute Effektivität mit einer Verbesserung der Lebensqualität kombiniert. So zeigte der Antimetabolit, der zielgerichtet in gleich drei Enzymsysteme der Zelle eingreift, in der Studie von Hanna et al. [3] im Vergleich zum
Abb. 1. Metanalyse belegt überlegene Effektivität von Platin-haltigenGemcitabin-Kombinationen beim NSCLC - modifiziert nach [2]. 
bisherigen Therapiestandard Docetaxel eine ebenso gute Effektivität. Die Verabreichung einer 10-minütigen Infusion von $500 \mathrm{mg} / \mathrm{m}^{2} \mathrm{Pe}$ metrexed, zuzüglich Folsäure und Vitamin B12, führte zu einem Gesamtüberleben von 8,3 Monaten. Nach einem Jahr lebten noch 30\% der Patienten. Die progressionsfreie Zeit betrug 14,6 Wochen. Hinsichtlich des Toxizitätsprofils schnitt Pemetrexed deutlich besser ab als das Vergleichsregime. Als hochsignifikant erwies sich das unterschiedliche Spektrum hämatologischer Nebenwirkungen, insbesondere für Neutropenien. So erlitten 40,2\% der mit Docetaxel behandelten Patienten eine schwere Neutropenie (Grad 3 und 4), unter Pemetrexed dagegen nur 5,3\%. Auch die Inzidenz des neutropenischen Fiebers war mit einer Rate von 1,9\% für Pemetrexed signifikant niedriger als für Docetaxel mit 12,7\%. Diese wesentlich geringeren Toxizitäten hatten deutliche Auswirkungen auf das Therapiemanagement und die Befindlichkeit der Patienten. So mussten unter Pemetrexed weniger Patienten in das Krankenhaus eingewiesen werden, und auch der Einsatz von Wachstumsfaktoren wurde vermindert.

\section{Mehr Lebensqualität durch weniger Toxizität}

Weitere Auswertungen der Toxizitätsdaten dokumentierten, dass die Patienten von Pemetrexed einen deutlichen Mehrwert gegenüber Docetaxel haben. In der detaillierten Analyse der Toxizitäten von Bhalla et al. [4] konnte für die Patienten der PemetrexedGruppe eine signifikant längere toxizitätsfreie Zeit als unter Docetaxel nachgewiesen werden (Tage ohne hämatologische Toxizität: 69,7 vs. $42,3, p<0,001$; Tage ohne nichthämatologische Toxizität 22,8 vs. $16,4, \mathrm{p}=$ 0,04). Daten von Pujol et al. [5] demonstrieren, dass Patienten aus der Pemetrexed-Gruppe eine signifikant kürzere Zeit unter Toxizitäten mit dem Schweregrad 3/4 litten als mit Docetaxel behandelte Patienten. «Das heißt, die verbleibende Lebensspanne der Patienten wird bei Pemetrexed nicht mit unnötigen Toxizitäten belastet und der Effekt der Therapie ist umso besser», so Hanauske. Auf Grund dieser ermutigenden Daten wird Pemetrexed in Kombination mit Cisplatin in der First-lineTherapie getestet. Entsprechende Studiendaten werden in Kürze erwartet (Abb. 2).

\section{Paradigmenwechsel beim Pleura- mesotheliom durch Pemetrexed}

«Bevor Pemetrexed verfügbar war, gab es für Mesotheliom-Patienten praktisch keine Hoffnung, sie verstarben meist binnen eines Jah-
Abb. 2. Signifikant längere toxizitätsfreie Zeit unter Pemetrexed - modifiziert nach [4].

res nach Diagnosestellung», so PD Dr. Martin Reck, Großhansdorf. Angesichts von 5000 jährlichen Neudiagnosen und stetig steigender Inzidenz des so genannten Asbesttumors ein unhaltbarer Zustand. Den Durchbruch brachte die so genannte EMPHACISStudie bei fast 450 Patienten mit einem malignen Pleuramesotheliom. In der bisher größten beim malignen Pleuramesotheliom durchgeführten Studie mit 448 Patienten konnte gezeigt werden, dass die Kombination Pemetrexed/Cisplatin (10-Minuten-Infusion Pemetrexed $500 \mathrm{mg} / \mathrm{m}^{2}$ plus Cisplatin $75 \mathrm{mg} /$ $\mathrm{m}^{2}$, Therapietag 1 alle 3 Wochen) gegenüber der Cisplatin-Monotherapie die mediane Überlebenszeit um fast 3 Monate verlängert (12,1 vs. 9,3 Monate). Unter der Kombination erreichten $41 \%$ der Patienten eine partielle Remission, unter der Monotherapie nur 17\% ( $\mathrm{p}<0,0001)$. Durch die Supplementierung von Vitamin B12 ließ sich die Remissionsrate von 41 auf $46 \%$ erhöhen. Nach einem Jahr lebten noch mehr als die Hälfte der Patienten aus dem Kombinationstherapie-Arm im Vergleich zu 38\% im Cisplatin-Arm. Die Zeit bis zur Progression betrug 5,7 vs. 3,9 Monate (p $=0,008$ ).

Neben einem verbesserten Ansprechen und einer Verlängerung der Überlebenszeit bewirkt die Behandlung mit Pemetrexed ebenfalls eine signifikant verbesserte Lebensqualität. Hier hob Reck eine Verringerung des Tumorschmerzes und eine erhöhte Lungenfunktion hervor. Diese äußerte sich in einer signifikant verbesserten Ruhevitalkapazität. Darüber hinaus verbesserten sich auch die Fatigue sowie Symptome wie Husten und Atemnot. Reck schlussfolgerte auf Grund dieser überzeugenden Daten: «Die Kombination von Pemetrexed mit Cisplatin stellt beim Pleuramesotheliom den Behandlungsstandard dar».

\section{Quelle}

Symposium «Therapie thorakaler Tumoren Aufbruch in eine neue Ära», DGHO, Leipzig, 5.11.2006.

\section{Referenzen}

1 Ardizzoni A, Tiseo M, Boni L, et al.: CISCA (cisplatin vs. carboplatin) meta-analysis: an individual patient data meta-analysis comparing cisplatin versus carboplatin-based chemotherapy in first-line treatment of advanced non-small cell lung cancer. Proc Am Soc Clin Oncol 2006;24:366s. abstr 7011. 2 Le Chevalier T: Efficacy of gemcitabine plus platinum chemotherapy compared with other platinum containing regimens in advanced nonsmall-cell lung cancer: a metaanalysis of survival outcomes. Lung Cancer 2005;47;69-80.

3 Hanna N, et al.: Randomized phase III trial of pemetrexed versus docetaxel in patients with nonsmall cell lung cancer previously treated with chemotherapy. J Clin Oncol 2004;22:1589-1597. 4 Bhalla S, et al.: Does reporting expected duration and severity of adverse events provide clinically relevant information when selecting a chemotherapy regimen? An example using pemetrexed and docetaxel. 10th World Conference on Lung Cancer 2005; abstr PD - 066.

5 Pujol J., et al.: Risk-benefit of pemetrexed compared with docetaxel in patients with advanced non-small cell lung cancer (NSCLC) previously treated with chemotherapy: An analysis of toxicityfree survival. Ann Oncol 2004;15(S3) Abstr. 795P.

\section{Bericht}

Bettina Reich, Hamburg.

\begin{tabular}{l} 
Impressum \\
\hline Gemcitabin und Pemetrexed bieten \\
verbesserte Perspektiven bei thorakalen \\
Tumoren \\
PharmaForum in ONKOLOGIE 30 | 1-2 | 07 \\
PM 041048 \\
C) 2007 by S. Karger Verlag für Medizin \\
und Naturwissenschaften GmbH \\
Lörracher Straße 16a \\
79115 Freiburg, Deutschland
\end{tabular}

Mit freundlicher Unterstützung von Lilly Deutschland GmbH.

Gemzar $^{\circledR}$ und Alimta ${ }^{\circledR}$ sind eingetragene Warenzeichen der Lilly Deutschland GmbH.

Der Verlag und die Herausgeber der Zeitschrift übernehmen keine Verantwortung für diese Rubrik.

\section{KARGER ๑) 2007 S. Karger GmbH, Freibur}

